\title{
Quality of Life in Patients with Osteoporotic Vertebral Fractures
}

\author{
Sang-Pil Yoon ${ }^{1}$, Seung-Hwan Lee ${ }^{1}$, Chul-Hyun $\mathrm{Ki}^{1}$, Young-Tae Lee ${ }^{1}$, \\ Sung-Ha Hong ${ }^{1}$, Hwan-Mo Lee ${ }^{2}$, Seong-Hwan Moon ${ }^{2}$ \\ ${ }^{1}$ Department of Orthopaedic Surgery, Kwang-Myung Sung-Ae Hospital, Seoul, Korea \\ ${ }^{2}$ Department of Orthopaedic Surgery, Yonsei University College of Medicine, Seoul, Korea
}

\begin{abstract}
Study Design: A case-control study.
Purpose: To examine several dimensions of health-related quality of life (HROL) in postmenopausal women with osteoporotic vertebral fractures, compared with a control group.

Overview of Literature: Osteoporotic vertebral fractures are a major cause of morbidity among postmenopausal women. There have been many reports of a decrease in the quality of life in patients with osteoporotic vertebral fractures. However,few reports have analyzed which dimensions contribute to the decline in quality of life.

Methods: One thousand five hundred forty-five postmenopausal women aged 50 years and older from 17 study sites in nationwide hospitals were in enrolled in the study (between April 2008 and January 2009). HROL was measured using the European Quality of Life 5 Domains (EQ-5D), and visual analogue scale (VAS).

Results: The average VAS of the case group was 57.80 , and that of the control group was $64.10(p=0.001)$. All domains of the EQ$5 \mathrm{D}$ score were significantly worse in the case group $(p=0.001)$. Among the case group, the average VAS of the 559 patients $(45 \%)$ who were operated on was 56.8 , and that of the remaining 680 patients $(55 \%)$ who were treated conservatively was $58.6(p=0.135)$. Among the case group, the averages of each EQ-5D domain of the 559 patients (45\%) who were operated on were: 1.87 in mobility, 1.81 in self-care, 1.99 in usual activities, 2.11 in pain, and 1.62 in anxiety or depression. Those of the 680 patients (55\%) who were treated conservatively were: 1.72 in mobility, 1.60 in self-care, 1.76 in usual activities, 1.98 in pain, and 1.57 in anxiety or depression. Except for the domain of anxiety or depression, scores for the other domains were all significantly worse in the patients who were operated on $(p=0.001)$.

Conclusions: Health related quality of life in the patients with osteoporotic vertebral fractures was significantly worse in both the EQ-5D domains and VAS. Among the osteoporotic vertebral fracture patients, the patients who were operated on had a worse quality of life in EQ-5D.
\end{abstract}

Keywords: Osteoporotic vertebral fracture; Health-related quality of life

\section{Introduction}

Osteoporotic vertebral fractures are a major clinical concern in postmenopausal women. Vertebral fractures are the most prevalent osteoporosis-related fractures .However, they are often asymptomatic, and their under-diagnosis and under-treatment has been well documented $[1,2]$. Vertebral fractures and their related deformities re-

Received Dec 22, 2013; Revised Feb 1, 2014; Accepted Feb 1, 2014

Corresponding author: Seong-Hwan Moon

Department of Orthopaedic Surgery, Yonsei University College of Medicine,

\#134 Shinchondong, Seodaemunku, Seoul, Korea

Tel: +82-2-2228-2188, Fax: +82-2-363-1139, E-mail: shmoon@yuhs.ac 
sult in back pain, disability, limitations in physical function, and psychosocial impairment [3].

There have been many reports in the literature of a decrease in the quality of life of patients with osteoporotic vertebral fractures $[2,4-8]$. In contrast few reports have analyzed which dimensions contribute to the decline in quality of life. The European Quality of Life 5 Dimensions Index (EQ-5D) is a generic, preference-based instrument that providesa comprehensive framework within which to determine health status and measure health-related quality of life (HRQL) $[9,10]$. The EQ-5D describes the state of health in 5 dimensions: mobility, self-care, usual activities, pain or discomfort, and anxiety or depression.

The purpose of our study was to examine the impact of osteoporotic vertebral fractures on each dimension of quality of life. Using data from 1,545 postmenopausal women aged 50 years and older from nationwide hospitals, we performed a case-control study to examine the HRQL using both EQ-5D and visual analogue scale (VAS) in postmenopausal women with osteoporotic vertebral fractures.

\section{Materials and Methods}

One thousand five hundred forty-five postmenopausal women aged 50 years and older from 17 study sites nationwide were asked to participate in this this study. Enrollment occurred between April 2008 and January 2009. The total number of patients in the case group who had osteoporotic vertebral fractures was 1,239, while 559 of these patients $(45 \%)$ were operated on using vertebroplasty, kyphoplasty, or spinal instrumentation. The total number in the control group, with no vertebral fracture, was 306 .

\section{Study population}

The patients who had osteoporosis (T-score below -2.5) and a morphometric vertebral fracture on the lateral thoracic and lumbar X-ray, and satisfying one of the categories below, were included in the case group: 1) Height loss of more than $2.5 \mathrm{~cm}$ during the last 1 year, 2) Local tenderness on the fracture site, 3 ) Evidence of a recent fracture on an magnetic resonance imaging (MRI) or whole body bone scan, 4) History of trauma during the past 3 months, 5) Surgery for a compression fracture during the past 3 months.
One thousand sixteen patients (82\%) among the case group had local tenderness at the site of the vertebral fracture; 495 patients (40\%) had evidence of a recent fracture on an MRI or whole body bone scan; 470 patients (38\%) had a history of trauma within 3 months (Table 1).

The control group consisted of patients with osteoporosis, without a history of fracture. The exclusion criteria were a history of cancer, spinal disorder, infection, and the inability to communicate.

\section{Measures analyzed}

Our main outcome, HRQL, was measured by the EQ5D scale and VAS. The EQ-5D is a 5-domain, 3-response option scale. On the 3-response option scale, level 1 was regarded as experiencing no problems in the EQ-5D domain, level 2 was regarded as having minor problems, and level 3 was regarded as having severe problems in that domain. Each of the possible 243 health states was mapped to a country-specific preference-based value or utility, in which 1.00 represents full health and 0.00 represents a state equivalent to death [11]. The EQ-5D index was calculated using this country-specific preferencebased value.

The VAS was measured as a score in which 0 represents the worst health status that the patient can imagine, and 100 represents the best health status. The EQ-5D scale and VAS was reported directly by the patient. 65 orthopaedic physicians participated in this study.

\section{Statistical analysis}

The EQ-5D dimensions were compared between the case group and the control group using a chi-square test and $t$-test. VAS was compared using a $t$-test.

\section{Results}

The mean age of the case group was 71.6, while mean of the control group was 67.7. The percentage of the absence of chronic disease was $43 \%$ in the case group, and $48 \%$ in the control group. Prevalence of hypertension was $37 \%$ in the case group and 33\% in control group, diabetes mellitus was $17 \%$ and $18 \%$, heart disease was $8 \%$ and $7 \%$, and arthritis was $5 \%$ and $4 \%$, respectively (Table 1 ).

A brace was applied on 1,127 patients (91\%) in the case group for 12 weeks. Pain killers were prescribed to 
Table 1. Descriptive data of case group and control group

\begin{tabular}{|c|c|c|c|}
\hline Characteristic & Case group $(1,239)$ & Control group (306) & $p$-value \\
\hline Age (yr) & $71.62( \pm 7.83)$ & $67.74( \pm 8.10)$ & 0.001 \\
\hline Bone mineral density $\left(\mathrm{mg} / \mathrm{cm}^{2}\right)$ & $0.57( \pm 0.17)$ & $0.61( \pm 0.16)$ & 0.018 \\
\hline \multicolumn{4}{|l|}{ Inclusion criteria } \\
\hline Local tenderness & $1,016(82)$ & & \\
\hline Evidence of recent fracture on MRI or whole body bone scan & $496(40)$ & & \\
\hline History of trauma within 3 months & $471(38)$ & & \\
\hline Height loss more than $2.5 \mathrm{~cm}$ during last 1 year & $173(14)$ & & \\
\hline Surgery for compression fracture during last 3 months & $99(8)$ & & \\
\hline \multicolumn{4}{|l|}{ Chronic disease } \\
\hline Hypertension & $458(37)$ & $101(33)$ & \\
\hline Diabetes mellitus & $210(17)$ & $55(18)$ & \\
\hline Heart disease & $99(8)$ & $21(7)$ & \\
\hline Arthritis & $62(5)$ & $12(4)$ & \\
\hline No chronic disease & $532(43)$ & $147(48)$ & \\
\hline
\end{tabular}

$\mathrm{MRI}$, magnetic resonance imaging.

Values are presented as mean \pm standard deviation or number (\%).

Table 2. Comparion of EQ-5D domains between case group and control group using chi-square test

\begin{tabular}{|c|c|c|c|}
\hline EQ-5D domains & Case group $(1,239)$ & Control group (306) & $p$-value \\
\hline Mobility & 886 (71.5) & 152 (49.7) & $<0.001$ \\
\hline Self-care & $725(58.5)$ & $94(30.7)$ & $<0.001$ \\
\hline Usual activities & $945(76.3)$ & $152(49.7)$ & $<0.001$ \\
\hline Pain & $1,081(87.2)$ & $224(73.2)$ & $<0.001$ \\
\hline Anxiety or depression & $659(53.2)$ & $123(40.2)$ & $<0.001$ \\
\hline
\end{tabular}

Values are presented as number (\%).

EQ-5D, Quality of Life 5 Domains.

1,177 patients (95\%) in the case group using non-opioids (paracetamol, nonsteroidal anti-inflammatory drugs, tramadol) and opioids.

For the individual problems of the EQ-5D domains, problems were detected in patients in the following rates: mobility problems in $71.5 \%$ of the case group and $49.7 \%$ of the control group $(p<0.001)$; self-care problems in $58.5 \%$ of the case group and $30.7 \%$ of the control group $(p<0.001)$; usual activities problems in $76.3 \%$ of the case group and $49.7 \%$ of the control group ( $p<0.001)$; pain in $87.2 \%$ of the case group and $73.2 \%$ of the control group $(p<0.001)$; anxiety or depression in $53.2 \%$ of the case group and $40.2 \%$ of the control group $(p<0.001)$. The mean EQ-5D index was 0.488 in the case group, and 0.701 in the control group $(p<0.001)$. The mean VAS was 57.8 in the case group, and 64.8 in the control group $(p<0.001)$ (Table 2).

The difference in the average VAS of the case group (57.80) and that of the control group (64.10) was statistically significant $(p=0.001)$. In the case group, the averages of each of the EQ-5D domains were as follows: 1.79 in mobility, 1.69 in self-care, 1.86 in usual activities, 2.03 in pain, and1.59 in anxiety or depression. The average of the control group were: 1.50 in mobility, 1.32 in self-care, 1.50 in usual activities, 1.76 in pain,and1.41 in anxiety or depression. All domains of the EQ-5D score were significantly worse in the case group ( $p=0.001$ ) (Table 3 ).

Operative treatment was performed on 559 patients (45\%) in the case group. Vertebroplasty was done on $51 \%$, balloon kyphoplasty was done on $45 \%$, and spinal 
Table 3. Comparison of EQ-5D and VAS between case group and control group using $t$-test

\begin{tabular}{lccc} 
EQ-5D dimensions & Case group (1,239) & Control group (306) & $p$-value \\
Mobility & $1.79( \pm 0.57)$ & $1.50( \pm 0.53)$ & 0.001 \\
Self-care & $1.69( \pm 0.66)$ & $1.32( \pm 0.50)$ & 0.001 \\
\hline Usual activities & $1.86( \pm 0.57)$ & $1.50( \pm 0.52)$ & 0.001 \\
Pain & $2.03( \pm 0.54)$ & $1.76( \pm 0.49)$ & 0.001 \\
Anxiety or depression & $1.59( \pm 0.61)$ & $1.41( \pm 0.52)$ & 0.001 \\
E0-5D index & $0.49( \pm 0.23)$ & $0.70( \pm 0.21)$ & 0.001 \\
VAS & $57.80( \pm 20.87)$ & $64.81( \pm 20.06)$ & 0.001 \\
\hline
\end{tabular}

Values are presented as mean \pm standard deviation.

EQ-5D, Quality of Life 5 Domains; VAS, visual analogue scale.

Table 4. Comparison of EQ-5D and VAS between operated patient and non-operated patient in case group using t-test

\begin{tabular}{lccc} 
EQ-5D domains & Operated case group (559) & Non-operated case group (680) & $p$-value \\
Mobility & $1.87( \pm 0.60)$ & $1.72( \pm 0.53)$ & 0.001 \\
Self-care & $1.81( \pm 0.69)$ & $1.60( \pm 0.62)$ & 0.001 \\
\hline Usual activities & $1.99( \pm 0.58)$ & $1.76( \pm 0.54)$ & 0.001 \\
Pain & $2.10( \pm 0.56)$ & $1.98( \pm 0.52)$ & 0.001 \\
Anxiety or depression & $1.62( \pm 0.63)$ & $1.57( \pm 0.58)$ & 0.155 \\
VAS & $56.79( \pm 22.62)$ & $58.63( \pm 19.30)$ & 0.135 \\
\hline
\end{tabular}

Values are presented as mean \pm standard deviation.

E0-5D, Quality of Life 5 Domains; VAS, visual analogue scale.

instrumented fusion was done on $6 \%$ of the cases. Relative risk of operative treatment was significantly higher in patients with a bone marrow density $\left(\mathrm{mg} / \mathrm{cm}^{2}\right)$ less than 0.55 ( $p<0.001$, odds ratio, 1.79; 95\% confidence interval, $1.30-2.42)$.

In the case group, the average VAS of the 559 patients (45\%) who received an operation was 56.8, while that of the 680 patients $(55 \%)$ who were treated conservatively was 58.6. The difference was not significant ( $p=0.135)$. Among the case group, the average of each of the EQ-5D domains of the group of patients who had received operation was as follows: 1.87 in mobility, 1.81 in self-care, 1.99 in usual activities, 2.11 in pain, and 1.62 in anxiety or depression. The averages of the group treated conservatively were: 1.72 in mobility, 1.60 in self-care, 1.76 in usual activities, 1.98 in pain, and 1.57 in anxiety or depression. Excluding the domain of anxiety or depression, the scores of all the other domains were significantly worse in the operated patients $(p=0.001)$ (Table 4$)$.

\section{Discussions}

This case-control study shows that postmenopausal women aged 50 years and older with a recent osteoporotic vertebral fracture have a lower HRQL than postmenopausal women without an osteoporotic vertebral fracture. Reduction of the EQ-5D index was approximately 0.21 compared to the control group, and slightly higher than a previously reported study [12]. The fact that our patient group consisted of patients with recent vertebral fractures reflects the relatively lower EQ-5D index compared to patients with remote fractures.

In the current study, scores of all the EQ-5D domains in the case group were significantly worse compared with the control group. Differences of scores in each domain were relatively high in mobility, self-care, and usual activities. In the case group, patients who were operated on had worse EQ-5D scores in all domains, except anxiety or depression. Conservative treatment of osteoporotic vertebral fractures showed to have more benefit in the quality 
of life of patients.

Back muscle strength and spinal mobility are also predictors of quality of life in postmenopausal women $[13,14]$. Patients with vertebral compression fractures usually have back muscle weakness and decreased spinal mobility because of prolonged immobilization with a body jacket cast or bed rest. These can cause difficulty in self-bathing or clothing, and a decrease in daily living in our study.

Pain is another common problem after vertebral compression fractures. In a recent study, one-third of the patients with vertebral compression fractures still had severe pain, necessitating pain medication and physical therapy [15]. However, no predictors for the transition from acute to chronic pain could be identified [16]. In our study, VAS was significantly higher in the case group, and risk of continuing pain was also higher compared to the control group.

Psychological problems often occur in patients with osteoporotic vertebral fractures. They express substantial anxiety, especially about the possibility of future fractures and physical deformity. As the disease progresses, depression can become a main problem [3]. In our study, the risk of anxiety and depressive problems was also high.

The strength of our study is that we enrolled case and control group patients with osteoporotic vertebral fracture who did not have a history of other fractures, so we could assess the impact of osteoporotic vertebral fractures on HRQL while controlling the impact of other fractures. This study has one of the larger population samples with both densitometric and spine X-ray evaluations. Assessment of vertebral fracture was carried out with standardized and reliable methods.

The major limitation of the current study was that since only patients with recent vertebral fractures were enrolled, we were unable to account for the effect of time lapse on the quality after the fracture occurred. If we had been able to collect the HRQL data after several decades, the effect of the fractures on quality of life would probably have been more pronounced.

Misclassification of comparable medical conditions is also possible, and would likely result in an underestimation of their effect on the quality of life. One reason for combining rheumatoid arthritis and osteoarthritis was the concern that many respondents who report the former may have the latter. Some patients with type 2 diabetes may have reported type 1 diabetes. If this was the case, the effect of diabetes on HRQL would likely be underestimated.

\section{Conclusions}

In this study, health related quality of life in the patients with osteoporotic vertebral fractures was significantly worse in the EQ-5D domains and VAS. Differences for the scores in each domain were relatively high in mobility, self-care, and usual activities. Among the osteoporotic vertebral fracture patients, those who were operated on had a worse quality of life displayed by EQ-5D. This suggests conservative treatment of osteoporotic vertebral fracture should be considered first when treating these patients, considering the aspect of quality of life.

\section{Conflict of Interest}

No potential conflict of interest relevant to this article was reported.

\section{References}

1. Delmas PD, van de Langerijt L, Watts NB, et al. Underdiagnosis of vertebral fractures is a worldwide problem: the IMPACT study. J Bone Miner Res 2005; 20:557-63.

2. Majumdar SR, Kim N, Colman I, et al. Incidental vertebral fractures discovered with chest radiography in the emergency department: prevalence, recognition, and osteoporosis management in a cohort of elderly patients. Arch Intern Med 2005;165:905-9.

3. Gold DT. The clinical impact of vertebral fractures: quality of life in women with osteoporosis. Bone 1996;18:185S-9S.

4. Hall SE, Criddle RA, Comito TL, Prince RL. A casecontrol study of quality of life and functional impairment in women with long-standing vertebral osteoporotic fracture. Osteoporos Int 1999;9:508-15.

5. Lips P, van Schoor NM. Quality of life in patients with osteoporosis. Osteoporos Int 2005;16:447-55.

6. Naves Diaz M, Diaz Lopez JB, Rodriguez Rebollar A, Gomez Alonso C, Diaz Corte C, Cannata Andia J. Effect of vertebral fracture on health related quality of life in a Spanish population older than 54 years. Med Clin (Barc) 2001;116:533-5.

7. Tosteson AN, Gabriel SE, Grove MR, Moncur MM, 
Kneeland TS, Melton LJ 3rd. Impact of hip and vertebral fractures on quality-adjusted life years. Osteoporos Int 2001;12:1042-9.

8. van Schoor NM, Smit JH, Twisk JW, Lips P. Impact of vertebral deformities, osteoarthritis, and other chronic diseases on quality of life: a population-based study. Osteoporos Int 2005;16:749-56.

9. Brazier JE, Walters SJ, Nicholl JP, Kohler B. Using the SF-36 and Euroqol on an elderly population. Qual Life Res 1996;5:195-204.

10. Brooks R. EuroQol: the current state of play. Health Policy 1996;37:53-72.

11. Sullivan PW, Lawrence WF, Ghushchyan V. A national catalog of preference-based scores for chronic conditions in the United States. Med Care 2005;43:736-49.

12. Adachi JD, Adami S, Gehlbach S, et al. Impact of prevalent fractures on quality of life: baseline results from the global longitudinal study of osteoporosis in women. Mayo Clin Proc 2010;85:806-13.
13. Imagama S, Matsuyama Y, Hasegawa Y, et al. Back muscle strength and spinal mobility are predictors of quality of life in middle-aged and elderly males. Eur Spine J 2011;20:954-61.

14. Miyakoshi N, Hongo M, Maekawa S, Ishikawa Y, Shimada Y, Itoi E. Back extensor strength and lumbar spinal mobility are predictors of quality of life in patients with postmenopausal osteoporosis. Osteoporos Int 2007;18:1397-403.

15. Klazen CA, Verhaar HJ, Lohle PN, et al. Clinical course of pain in acute osteoporotic vertebral compression fractures. J Vasc Interv Radiol 2010;21:14059.

16. Venmans A, Klazen CA, Lohle PN, Mali WP, van Rooij WJ. Natural history of pain in patients with conservatively treated osteoporotic vertebral compression fractures: results from VERTOS II. AJNR Am J Neuroradiol 2012;33:519-21. 